\title{
Elucidation of the Multiple Activities of Abiraterone by a Synthetic Chemistry
} Approach

\author{
Miaoling He${ }^{1}$, Ben Yi Tew ${ }^{1}$, Stein CY ${ }^{2}$, Darryl Rideout ${ }^{3}$, Sumanta K Pal ${ }^{2}$ and Jeremy O Jones ${ }^{1^{*}}$
}

${ }^{1}$ Department of Cancer Biology, City of Hope Beckman Research Institute, Duarte, CA, USA

${ }^{2}$ Department of Medical Oncology and Experimental Therapeutics, City of Hope Comprehensive Cancer Center, Duarte, CA, USA

${ }^{3}$ Focus Synthesis LLC, San Diego, CA, USA

\begin{abstract}
Objective: Novel agents to treat metastatic prostate cancer include a class of drugs which function primarily by inhibiting the action of the CYP17 enzyme, which results in decreased androgen synthesis. Abiraterone and related molecules have been shown to decrease expression of the androgen receptor $(A R)$ and to inhibit its transcriptional activity in addition to CYP17 inhibition. The structure-activity relationships (SAR) governing AR down regulation are less well understood than CYP17 and AR inhibition, so we designed abiraterone derivatives with the goal of better understanding the SAR of AR down regulation.
\end{abstract}

Methods: We synthesized 17 abiraterone derivatives with unsaturated, cyclic and acyclic substituents containing hydrogen bond acceptors at $\mathrm{C} 16$ and $\mathrm{C} 17$ positions. We examined the ability of these compounds to inhibit CYP17 and $A R$, and to decrease $A R$ expression.

Results: While abiraterone was the most potent AR down regulator, we found that a hydrogen bond acceptor on an unsaturated nitrogen or oxygen, 4 to 6 angstroms from $\mathrm{C} 17$ is required for AR down-regulation, but a heterocyclic ring is not necessary, as shown by the activity of compounds 4,8 , and 10 . The size and shape of substituents on active AR down regulators indicates that the binding site near $\mathrm{C} 17$ is significantly larger than the pyridine ring in abiraterone. We found no correlation between the ability of a compound to down regulate AR and its ability to inhibit CYP17 or AR transcriptional activity. AR transcriptional activity was inhibited most potently by molecules with hydrogen bond donors 2-3 angstroms from C17. Molecules with C16 substituents could inhibit AR transcription but were inactive as AR down regulators, as indicated by compounds 5,13 , and 14 . CYP17 inhibitors had an unsaturated nitrogen (pyridine or hydrazine) oriented away from the steroid backbone and perpendicular to the $\mathrm{C} 16-\mathrm{C} 17$ bond.

Conclusion: We determined the SAR for AR down regulation. The complete lack of correlation between SARs for $A R$ down regulation and AR or CYP17 inhibition suggests distinct mechanisms of actions for these activities. The SAR for AR down regulation suggests that additional hydrogen bond acceptor side chains 4-6 angstroms from C17 could enhance the activity of abiraterone.

Keywords: CYP17 inhibitor; Abiraterone; Prostate cancer; Androgen receptor

\section{Introduction}

Despite incredible advances in detection and treatment, prostate cancer remains the second leading cause of cancer death in American men [1]. Signaling by the androgen receptor (AR) controls normal prostate growth and homeostasis but also drives the proliferation of malignant prostate cells. In the setting of metastatic prostate cancer, removal of testicular androgens by surgical or chemical castration initially leads to cancer regression; however, new tumors almost universally recur despite very low levels of systemic androgens and most of these castration-resistant prostate cancers (CRPCs) remain dependent on AR function for growth $[2,3]$. Several molecular mechanisms have been described to account for continued AR signaling in CRPC, including the amplification of AR [2], gain-of-function mutations in AR that confer greater sensitivity to androgens or increased recruitment of AR coactivator proteins [4], LBD-independent $\mathrm{N}$-terminal activation by growth factors, neuropeptides, and inflammatory mediators [57], expression of constitutively active AR splice variants (ARv's) [811], tumoral conversion of adrenal androgens [12], and intratumoral androgen production [13]. These basic discoveries have led to a renewed interest in AR as a target in CRPC and have led to the development of several new drugs that block the AR/androgen signaling axis. The first to gain FDA approval was the CYP17 inhibitor abiraterone acetate, which prevents androgen synthesis from adrenal glands and cancer tissue (5). Several other compounds that target androgen synthesis are in advanced clinical trials, including orteronel and galeterone [14,15]. In addition to inhibiting androgen synthesis, several CYP17 inhibitors been reported to decrease AR levels [15,16], including the levels of ARv's [17]. The decreased AR expression appears to occur through a suppression of cap-dependent translation by blocking assembly of the eIF4F and eIF4G complex to the mRNA 5' cap [16]. Several CYP17 inhibitors also reduce AR transcriptional activity, likely through competitive antagonism of ligand binding [16,17]. Decreasing AR levels could provide a valuable strategy for inhibiting the growth of prostate cancers, especially when combined with androgen synthesis inhibition and competitive antagonism. In an attempt to understand what chemical moieties control the various pharmacologic actions of these multi-activity drugs, we modified the structure of abiraterone and examined the ability of the derivatives to decrease AR expression and inhibit AR transcriptional activity as our main objectives. The ability of these molecules to inhibit testosterone synthesis through CYP17 inhibition was also determined as a secondary objective.

*Corresponding author: Jeremy O Jones, Beckman Research Institute City of Hope Comprehensive Cancer Center 1500 East Duarte Road Duarte, CA 91010, Tel: +626 256-4673; Fax: (626) 301-8233; E-mail: jjones@coh.org

Received: February 20, 2015; Accepted: April 15, 2015; Published: April 20, 2015

Citation: He M, Tew BY, Stein CY, Rideout D, Pal SK, et al. (2015) Elucidation of the Multiple Activities of Abiraterone by a Synthetic Chemistry Approach. Clin Exp Pharmacol 5: 173. doi:10.4172/2161-1459.1000173

Copyright: () $2015 \mathrm{He} \mathrm{M}$, et al. This is an open-access article distributed under the terms of the Creative Commons Attribution License, which permits unrestricted use, distribution, and reproduction in any medium, provided the original author and source are credited. 


\section{Results and Discussion}

Design strategy and chemistry: In this study, 17 novel derivatives were synthesized around the abiraterone (1) core with the goal of identifying moieties that affect the ability of abiraterone to inhibit CYP17 activity, down-regulate AR expression, and inhibit AR transcriptional activity. The modifications involved replacing the 17aryl group and the 16-hydrogen with other substituents in both 16-17 unsaturated and 16-17 saturated molecules (Figure 1). The substituents were chosen to better understand the SAR, specifically how the shape of substituents and position of hydrogen bond donors and acceptors affected AR down regulation activity.

\section{AR down-regulation}

Abiraterone has been shown to decrease both AR protein and mRNA levels, likely by interfering with cap-dependent translation [16]. To test the ability of derivatives to down- regulate AR expression, LNCaP prostate cancer cells were treated with each derivative at concentrations ranging from 0.5 to $25 \mathrm{uM}$ for $48 \mathrm{hrs}$ and AR was detected in lysates by Western blot. $48 \mathrm{hrs}$ was determined to be the optimal time-point for assay from pilot time-course studies with abiraterone (1). Derivatives were ranked in order of their ability to decrease AR protein levels compared to a vehicle control (Figure 1A). Abiraterone (1) and its 4 -isomer (2) were the most potent, but the activities of 8,11 , and 18 show that an aromatic ring is not necessary for activity. A hydrogen bond acceptor on an unsaturated $\mathrm{CN}$ or CO bond, 4 to 6 angstroms from C17 is a common feature for $1,2,8,9,10,11$, and 18 that is lacking in the weakly active 6 and inactive molecules 5, 14, 15, and 16. The importance of this hydrogen bond acceptor is further supported by the substantially greater AR down regulation activity of 8 compared to 12 and 13. Compound $8(\mathrm{C}=\mathrm{N}$ stereochemistry unknown $)$ is isosteric with either 12 or 13, differing in that 8 is a carbamoylhydrazone while 12 and 13 are alkanoylhydrazones. Compound 3 has an oxygen atom adjacent to the carbonyl, making the carbonyl in 3 more electronegative than the carbonyls in 12 and 13. Structures 9 and 10 also have more electron rich carbonyl groups and great AR down regulation activity than 12 and 13. Compound 2, with an electron-poor aroylhydrazone carbonyl and a pyridine nitrogen $>7$ angstroms from $\mathrm{C} 17$, is completely inactive. The maintenance of activity in molecules with large $\mathrm{C} 17$ groups $(7,10$, and 11) all demonstrate that the binding pocket occupied by the pyridyl ring in abiraterone is significantly larger than pyridyl, but does not extend as far as the pyridine ring in the inactive compound 2. Molecules with C16 substituents were inactive. The results suggest the possibility of designing molecules with greater potency than abiraterone by adding additional hydrophobic and hydrogen- bond acceptor groups to the aromatic ring.

\section{AR transcriptional activity}

$\mathrm{AR}$ is a transcription factor and abiraterone is an inhibitor of transcriptional activity as well as a down regulator of AR. We employed a firefly luciferase reporter driven by the human PSA promoter to assess the effect of derivatives on AR transcriptional activity in LNCaP cells. Although AR transcriptional activity inhibition and AR down regulation involve the same receptor, there is no correlation between these activities (Figures $1 \mathrm{~B}$ and $1 \mathrm{D}$ ), suggesting that very distinct mechanisms are responsible for AR transcriptional activity inhibition and AR down regulation. The oxime 6 is the most potent inhibitor of AR transcriptional activity $\left(\mathrm{IC}_{50}=0.1 \mathrm{uM}\right)$, but one of the least potent AR down regulators. Compounds 5, 13, and 14 are potent inhibitors of $\mathrm{AR}$ transcription $\left(\mathrm{IC}_{50}<1 \mathrm{uM}\right)$, but lack $\mathrm{AR}$ down regulatory activity.
Compound 8 is one of the least potent AR transcriptional inhibitors $\left(\mathrm{IC}_{50}=6.5 \mathrm{uM}\right)$, but is the 3rd most potent $\mathrm{AR}$ down regulator. The potent $A R$ transcriptional inhibition of $6,10,11,12,13\left(\mathrm{IC}_{50}<0.6 \mathrm{uM}\right)$, when contrasted with the weak activity of $18\left(\mathrm{IC}_{50}>8 \mathrm{uM}\right)$ suggest that a hydrogen bond donor within 2-3 Angstroms of $\mathrm{C} 17$ is consistent with activity. An electron-deficient aromatic ring $(1,4)$ or halogens (5) at $\mathrm{C} 17$ are also consistent with $\mathrm{AR}$ transcriptional inhibition. Bulky substituents well tolerated at both $\mathrm{C} 17\left(5,10,11\right.$, with $\mathrm{IC}_{50}<0.8$ $\mathrm{uM})$ and $\mathrm{C} 16\left(14,15,16,17\right.$ with $\left.\mathrm{IC}_{50}<2.3 \mathrm{uM}\right)$. However, very large, rigidly attached $\mathrm{C} 17$ substituents lead to much weaker transcriptional inhibition ( 2 and 7 , with $\mathrm{IC}_{50}>9 \mathrm{uM}$ ).

\section{CYP17 inhibition}

Although the structures of molecules used in this study were designed or selected to understand the structure/activity relationship for AR down regulation, we were also interested in CYP17 activity because it contributes to the therapeutic utility of abiraterone by inhibiting androgen synthesis. To determine the ability of derivatives to inhibit CYP17 enzymatic activity, we employed the NCI-H295R cell assay, a well-established model of steroidogenesis $[18,19]$. These cells express CYP17 and have been shown to synthesize $\mathrm{T}$ from steroidal precursors, which can be blocked by specific inhibitors [20]. Media was changed and cells were treated with $10 \mathrm{uM}$ of each compound for $48 \mathrm{hrs}$, at which point, $\mathrm{T}$ was quantified in the media using an ELISA specific for T (Figure 1C). The most active CYP17 inhibitors ( $>29 \%$ inhibition at $10 \mathrm{uM}$ ) contain an unsaturated $\mathrm{C}=\mathrm{N}$ bond, either in a pyridine ring nitrogen $(1,4)$ or a hydrazone with $\mathrm{Z}$ stereochemistry $(10,12)$. A loan pair oriented perpendicular to the $\mathrm{C} 16-\mathrm{C} 17$ bond and pointed away from the steroid framework is important: Z- hydrazones 10 and 12 (32 to $47 \%$ inhibition $10 \mathrm{uM}$ ) are more potent than E-isomers 9,11 and 13 ( 0 to $20 \%$ inhibition at $10 \mathrm{uM}$ ). For the $\mathrm{E}$ isomers, the nitrogen loan pair on $\mathrm{C}=\mathrm{N}$ is oriented parallel to the $\mathrm{C} 16-\mathrm{C} 17$ bond as opposed to perpendicular for the $\mathrm{Z}$ isomers. These results are in line with previous findings which suggest that CYP17 inhibition in abiraterone and related molecules depends upon an appropriately oriented heteroatom [21,22].

\section{Materials and Methods}

\section{Chemical syntheses}

Hydrazones 10, 11, 12 and 13 were synthesized using an established procedure (27). 16-Dehydropregnenolone was purchased from AK Scientific. Butyric acid hydrazide, Benzyl carbazate, and t-Butyl carbazate were purchased from Combi-Blocks, San Diego, CA, USA

\section{Z-16-Dehydropregnenolone butyroylhydrazone (12) and} E-16-Dehydropregnenolone butyroylhydrazone (13)

16-Dehydropregnenolone $(115 \mathrm{mg}, 0.366 \mathrm{mmol})$ was suspended in HOAc (1.15 ml). Butyric acid hydrazide $(133 \mathrm{mg}, 1.30 \mathrm{mmol}, 3.56$ equivalents) was added, and the mixture which was stirred at 25 degrees for 3 days. The mixture became homogenous during the reaction period. TLC (100\% diethyl ether) showed two products, Rf 0.30 (which formed quickly), and Rf 0.15 (which formed more slowly). Water (3 $\mathrm{ml}$ ) was added to the resulting yellow solution, and the precipitate was collected by centrifugation, then rinsed again with water $(3 \mathrm{ml})$, and chromatographed on silica gel with EtOAc- Hexanes (75:25) to afford the less polar product (12, with Rf 0.30 in diethyl ether) as a white gum (11 $\mathrm{mg}, 8 \%$ yield). The more polar product $(13, \mathrm{Rf}=0.15$ in diethyl ether) was isolated as a white solid (11 mg, $8 \%$ yield).

\section{Z-16-Dehydropregnenolone enzyloxycarbonylhydrazone (10)}

16- Dehydropregnenolone (102 $\mathrm{mg}, 0.32 \mathrm{mmol}$ ) was suspended in 


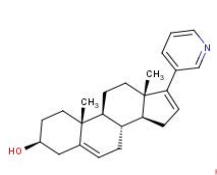

1 Abiraterone
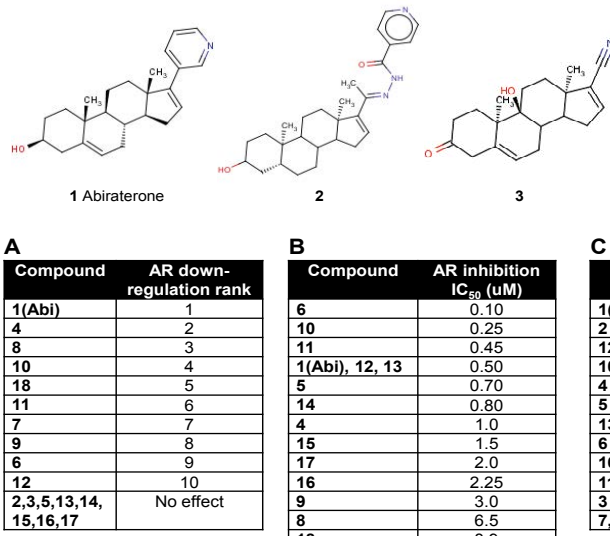

3
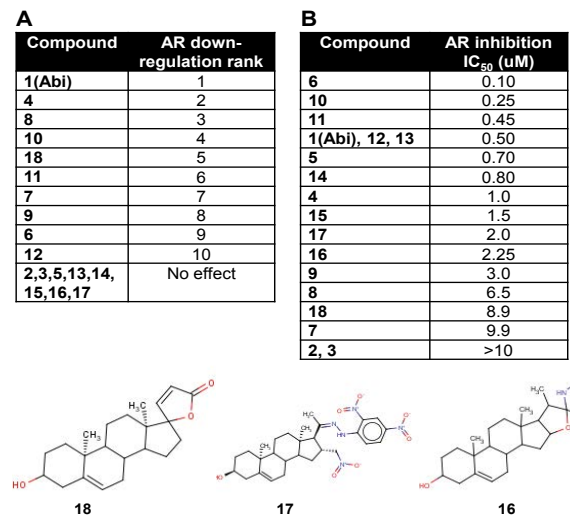

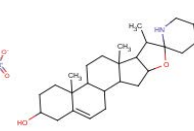

16
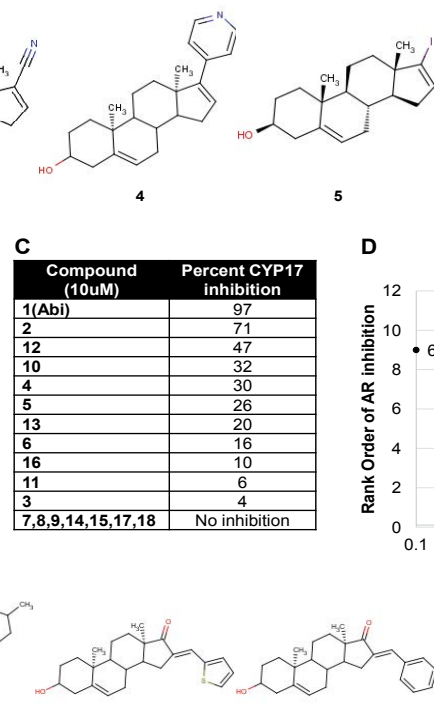

D
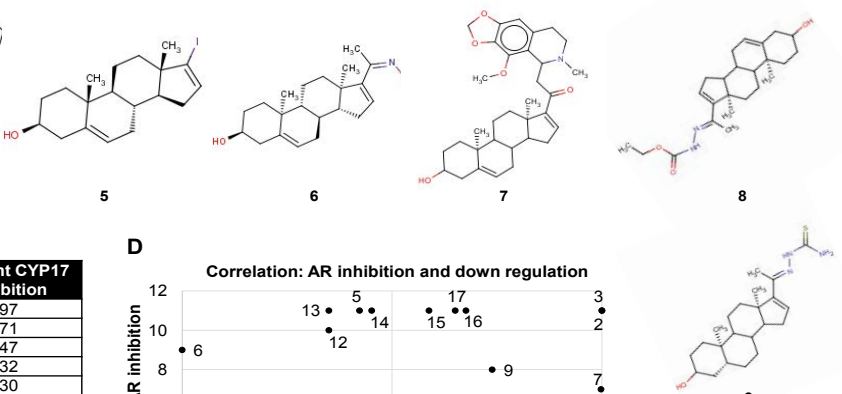

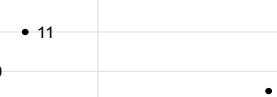

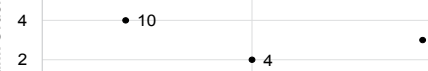

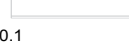

IC50 AR inhibition (UM)$$
\text { (1) }
$$

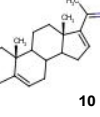

10

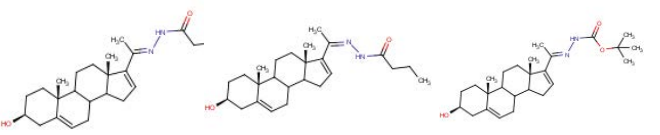

12

Figure 1: Activities of abiraterone derivatives: Structures of each compound are shown. (A) AR down regulation was determined by Western blot for AR expression and compounds were ranked by their ability to decrease AR expression. (B) The ability to inhibit AR transcriptional activity was determined by androgen-dependent luciferase assay, and compounds were ranked by IC . (C) CYP17 inhibition was determined by quantifying testosterone production by ELISA in H295R cells, and compounds were ranked by their ability to inhibit testosterone production compared to vehicle treated cells. (D) The lack of correlation between AR down regulation and inhibition of AR activity is demonstrated.

HOAc ( $1 \mathrm{ml})$. Benzyl carbazate $(102 \mathrm{mg}, 0.61 \mathrm{mmol}, 1.92$ equivalents) was added, and the mixture was stirred at 25-30 degrees for $1.75 \mathrm{~h}$. The mixture initially became homogenous, and then the product precipitated and was collected by vacuum filtration, rinsed with HOAc, and dried in vacuo to afford a white solid ( $40 \mathrm{mg}, 27 \%$ yield). TLC in diethyl ether showed a single spot at Rf 0.4. The melting point was 220 -222 degrees.

\section{E-16-Dehydropregnenolonet-butyloxycarbonylhydrazone} (10)

16- Dehydropregnenolone (141 $\mathrm{mg}, 0.45 \mathrm{mmol}$ ) was suspended in HOAc ( $1.15 \mathrm{ml}$ ). t-Butyl carbazate ( $141 \mathrm{mg}, 1.07 \mathrm{mmol}, 237 \mathrm{~mol} \%$ ) was added, and was stirred at 30 degrees for 4 days. During the reaction the mixture became homologous and turned yellow. Water $(3 \mathrm{ml})$ was added and the ppt was collected by centrifugation, then rinsed again with water $(3 \mathrm{ml})$, and chromatographed on silica gel with EtOAcHexanes (75:25) to afford the product as an off-white gum (40 mg, 21\% yield). TLC (EtOAc-Pet Ether, 75:25) showed a single spot with an Rf of 0.35 .

\section{Testosterone synthesis assay}

Human adrenal NCI-H295R cells (ATCC) were cultured in six well plates under standard conditions in Dulbecco's modified Eagle's/Ham's F-12 medium supplemented with 5\% Nu-Serum, $0.1 \%$ selenium/insulin/transferrin plus, and pen/strep. Twenty-four hours after subculturing, medium was replaced, and drugs were added at 10 $\mu \mathrm{M}$ in normal growth medium for $48 \mathrm{~h}$ to triplicate samples. Control cells were treated with $0.1 \%(\mathrm{v} / \mathrm{v})$ DMSO vehicle. $100 \mathrm{ul}$ of media was removed and processed for testosterone quantification according to the manufacturer's instructions (Diagnostic Automation Inc, Calabasas, $\mathrm{CA})$. The colorimetric assay was quantified on a M1000 Infinity plate reader (Tecan), and testosterone concentrations estimated by use of a standard curve. Percent inhibition was calculated compared to the vehicle control.

\section{Western blotting}

LNCaP cells were cultured under standard conditions with phenol red-free RPMI supplemented with 10\% FBS and pen/strep. Cells were treated with drugs for $4-72 \mathrm{~h}$ and then lysed in TBS with $0.1 \%$ Tween with protease inhibitors. Lysates were resolved by SDS-PAGE, transferred, and Western blot was performed with an anti-AR antibody (PG21, Millipore) or anti-GAPDH antibody (Santa Cruz, cat no. 47724) as a loading control.

AR transcription assay: Two days prior to transfection, LNCaP cells were placed in media containing charcoal-stripped serum. For all transfections, pools of cells were transfected using Lipofectamine Plus (Invitrogen) with PSA-luciferase and pRL-SV40 (Promega) as a control. The following day, the cells were plated in quadruplicate with drug [23] treatments in 96 well plates. $24 \mathrm{~h}$ later luciferase production was measured using the Dual luciferase assay kit (Promega) on the M1000 Infinity plate reader. Firefly signal was normalized to control Renilla luciferase signal and $\mathrm{IC}_{50}$ values were determined by linear regression.

\section{Conclusions}

AR down regulation requires a hydrogen bond acceptor on an unsaturated $\mathrm{N}$ or $\mathrm{O}, 4$ to 6 angstroms from $\mathrm{C} 17$, while for inhibition of AR transcription, molecules with hydrogen bond donors 2 to 3 angstroms from $\mathrm{C} 17$ can be more potent than abiraterone. The complete lack of correlation between SARs for AR down regulation and inhibition of AR transcription suggest distinct mechanisms of actions for these 
Citation: He M, Tew BY, Stein CY, Rideout D, Pal SK, et al. (2015) Elucidation of the Multiple Activities of Abiraterone by a Synthetic Chemistry Approach. Clin Exp Pharmacol 5: 173. doi:10.4172/2161-1459.1000173

2 activities (Figure 1D). Further synthesis will be necessary to dissect these functions. While no derivatives were synthesized with a greater ability than abiraterone to down regulate AR expression or inhibit CYP17, our studies will inform the synthesis of the next generation of derivatives with improved functionality. In particular, our SAR for AR down regulation suggests that the binding site for the aromatic sidegroup in abiraterone can accept larger molecules, such that a properly positioned hydrogen bond acceptor (e.g. carbamate) could enhance the activity of abiraterone.

\section{References}

1. Jemal AR, Siegel J, Xu Ward E (2010) Cancer statistics. CA Cancer J Clin 60: 277-300.

2. Chen CD, Welsbie DS, Tran C, Baek SH, Chen R, et al. (2004) Molecular determinants of resistance to antiandrogen therapy. Nat Med 10: 33-39.

3. Taplin ME, Balk SP (2004)Androgen receptor: a key molecule in the progression of prostate cancer to hormone independence. J Cell Biochem 91: 483-490.

4. Brooke GN, Parker MG, Bevan CL (2008) Mechanisms of androgen receptor activation in advanced prostate cancer: differential co-activator recruitment and gene expression. Oncogene 27: 2941-2950.

5. Araki S, Omori Y, Lyn D, Singh RK, Meinbach DM, et al. (2007) Interleukin-8 is a molecular determinant of androgen independence and progression in prostate cancer. Cancer Res 67: 6854-6862.

6. Debes JD, Schmidt LJ, Huang H, Tindall DJ ( 2002) p300 mediates androgenindependent transactivation of the androgen receptor by interleukin 6 . Cancer Res 62: 5632-5636.

7. Yeh S, Lin HK, Kang HY, Thin TH, Lin MF, et al. ( 1999) From HER2/Neu signal cascade to androgen receptor and its coactivators: a novel pathway by induction of androgen target genes through MAP kinase in prostate cancer cells. Proc Natl Acad Sci U S A 96: 5458-5463.

8. Dehm SM, Schmidt LJ, Heemers HV, Vessella RL, Tindall DJ (2008) Splicing of a novel androgen receptor exon generates a constitutively active androgen receptor that mediates prostate cancer therapy resistance. Cancer Res 68: 5469-5477.

9. Guo Z, Yang X, Sun F, Jiang R, Linn DE, et al. (2009) A novel androgen receptor splice variant is up-regulated during prostate cancer progression and promotes androgen depletion-resistant growth. Cancer Res 69: 2305-2313.

10. Hu R, Dunn TA, Wei S, Isharwal S, Veltri RW (2009) Ligand-independent androgen receptor variants derived from splicing of cryptic exons signify hormone-refractory prostate cancer. Cancer Res 69: 16-22.

11. Sun S, Sprenger CC, Vessella RL, Haugk K, Soriano K (2010) Castration resistance in human prostate cancer is conferred by a frequently occurring androgen receptor splice variant. J Clin Invest 120: 2715-2730.

12. Mostaghel EA, Montgomery B, Nelson PS (2009) Castration-resistant prostate cancer: Targeting androgen metabolic pathways in recurrent disease. Urol Oncol 27: 251- 257.

13. Knudsen KE, Penning TM (2010) Partners in crime: deregulation of AR activity and androgen synthesis in prostate cancer.Trends Endocrinol Metab 21: 315-324.

14. Kaku T, Hitaka T, Ojida A, Matsunaga N, Adachi M, et al. (2011) Discovery of orteronel (TAK-700), a naphthylmethylimidazole derivative, as a highly selective 17,20-lyase inhibitor with potential utility in the treatment of prostate cancer. Bioorg Med Chem 19: 6383-6399.

15. Bruno RD, Vasaitis TS, Gediya LK, Purushottamachar P, Godbole AM, et al. (2011) Synthesis and biological evaluations of putative metabolically stable analogs of VN/124-1 (TOK-001): head to head anti-tumor efficacy evaluation of VN/124-1 (TOK-001) and abiraterone in LAPC-4 human prostate cancer xenograft model. Steroids 76: 1268-1279.

16. Soifer HS, Souleimanian N, Wu S, Voskresenskiy AM, Collak FK, et al. (2012) Direct regulation of androgen receptor activity by potent CYP17 inhibitors in prostate cancer cells. J Biol Chem 287: 3777-3787.

17. Purushottamachar P, Godbole AM, Gediya LK, Martin MS, Vasaitis TS, et al (2013) Systematic structure modifications of multitarget prostate cancer drug candidate galeterone to produce novel androgen receptor down-regulating agents as an approach to treatment of advanced prostate cancer. J Med Chem 56: 4880-4898.

18. Gazdar AF, Oie HK, Shackleton CH, Chen TR, Triche TJ, et al. (1990) Establishment and characterization of a human adrenocortical carcinoma cel line that expresses multiple pathways of steroid biosynthesis. Cancer Res 50: 5488-5496.

19. Staels B, Hum DW, Miller WL (1993) Regulation of steroidogenesis in $\mathrm{NCl}-\mathrm{H} 295$ cells: a cellular model of the human fetal adrenal. Mol Endocrinol 7: 423-433.

20. Kempna P, Hofer G, Mullis PE, Fluck CE (2007) Pioglitazone inhibits androgen production in NCl-H295R cells by regulating gene expression of CYP17 and HSD3B2. Mol Pharmacol 71: 787-798.

21. Njar VC, Kato K, Nnane IP, Grigoryev DN, Long BJ, et al. (1998) Novel 17-azoly steroids, potent inhibitors of human cytochrome 17 alpha-hydroxylase-C17,20lyase (P450(17) alpha): potential agents for the treatment of prostate cancer. $J$ Med Chem 41: 902- 912.

22. Njar VC, Hector M, Hartmann RW (1996) 20-amino and 20,21-aziridinyl pregnene steroids: development of potent inhibitors of 17 alpha-hydroxylase/ C17,20-lyase (P450 17). Bioorg Med Chem 4: 1447-1453.

23. Bolton EC, So AY, Chaivorapol C, Haqq CM, Li H, et al. (2007) Cell- and genespecific regulation of primary target genes by the androgen receptor. Genes Dev 21: 2005-2017. 